\title{
Erratum di: Un commento a Boyers et al.: "Eltrombopag per il trattamento della porpora trombocitopenica immune o idiopatica cronica: valutazione di tecnologia singola presso il NICE"
}

Rachel Allen • Andres Brainsky • Kelly Grotzinger •

Tito Roccia

Pubblicato online: 5 marzo 2014

(C) Springer International Publishing Switzerland 2014

Erratum di: PharmacoEcon Ital Res Artic (2013)

15:153-155

DOI 10.1007/s40276-013-0014-2

Questo articolo è una Traduzione dell'articolo: A Comment on Boyers et al:: "Eltrombopag for the Treatment of Chronic Immune or Idiopathic Thrombocytopenic Purpura: A NICE Single Technology Appraisal" Pharmacoeconomics. 2013 January; 31(1): 87-89. DOI 10.1007/s40273-012-0003-8.

La versione online dell' articolo originale può essere trovata al doi:10.1007/s40276-013-0014-2.

R. Allen $(\bowtie) \cdot$ A. Brainsky $\cdot$ K. Grotzinger $\cdot$ T. Roccia

GlaxoSmithKline Ltd, 1-3 Iron Bridge Road, Stockley Park West,

Uxbridge UB11 1BT, UK

e-mail: rachel.1.allen@gsk.com 